\title{
A finite difference scheme for magneto-thermo analysis of an infinite cylinder
}

\author{
Daoud S. Mashat
}

Department of Mathematics, Faculty of Science, King AbdulAziz University, Jeddah, Saudi Arabia; dmashat@kau.edu.sa

Received 12 July 2010; revised 15 August 2010; accepted 20 August 2010.

\begin{abstract}
A finite different scheme as well as least-square method is presented for the magneto-thermo analysis of an infinite functionally graded hollow cylinder. The radial displacement, mechanical stresses and temperature as well as the electromagnetic stress are investigated along the radial direction of the cylinder. Material properties are assumed to be graded in the radial direction according to a novel exponential-law distribution in terms of the volume fractions of the metal and ceramic constituents. The governing second-order differential equations are derived from the equations of motion and the heat-conduction equation. The system of differential equations is solved numerically and some plots for displacement, radial stress, and temperature are presented.
\end{abstract}

Keywords: Infinite Cylinder;

Electro-Magneto-Thermoelastic; Finite Difference Method

\section{INTRODUCTION}

Magneto-thermal deformations of a cylinder can occur when the cylinder is placed in a constant primary magnetic field or due to heat exchange with the external or internal environments, or they can appear as the result of the deformations themselves, when part of the mechanical energy changes into heat [1-5]. Due to the complexity of the governing equations and the mathematical difficulties associated with the solution, several simplifications have been used. Yang and Chen [6] discussed the transient response of one-dimensional quasi-static coupled thermo-elasticity problems of an infinitely long annular cylinder composed of two different materials. They applied the Laplace transform with respect to time and used the Fourier series and matrix operations to obtain the solution. Jane and Lee [7] considered the same problem by using the Laplace transform and the finite difference method. The cylinder is composed of multilayers with different materials. There is no limit of number of annular layers of the cylinder in the computational procedures. Lee [8] presented axisymmetric quasi-static-coupled thermoelastic problems for timedependent boundary condition. Laplace transform and finite difference methods are used to analyze problems.

Using finite difference method, Awaji and Sivakuman [9] studied the transient thermal stresses of a FGM hollow circular cylinder, which is cooled by surrounding medium. A hybrid numerical method of the Laplace transformation and the finite difference is applied by Yang et al. [10] to solve the transient hygrothermal problem of an infinitely long annular cylinder, in which the temperature and moisture coupling at the inner and outer surfaces is taken into account in the boundary conditions. Chen [11] evaluated the tress intensity factors in a cylinder with a circumferential crack by using the finite difference method. Jane and Lee [12] considered the thermoelastic transient response of multilayered annular cylinders of infinite lengths subjected to known temperatures at traction-free inner and outer surfaces. A method based on the Laplace transformation and finite difference method has been developed to analyze the thermo-elasticity problem.

The primary objective of this investigation is to generate displacement, stresses, temperature, and magnetic field in an infinite FGM hollow cylinder. The present FGM cylinder is placed in a constant primary magnetic field. It is made of an isotropic material with material properties varying in the radial thickness direction only according to a novel power law form. The governing partial differential equations are obtained in conservation forms and solved numerically using finite difference method. Numerical results for the variation of temperature, displacement and stresses are presented for a metal-ceramic FG cylinder. To make the study reasonably, temperature, displacement, and stresses are distributed along the 
radial direction of the cylinder.

\section{MATHEMATICAL MODEL}

Let us consider a long cylinder of outer radius $r=b$, inner radius $r=a$, and made of an exponentially graded material. The cylindrical coordinates system $(r, \theta, z)$ is used with $z$-axis coinciding with the axis of the cylinder. The strain axis is considered to be symmetric about the $z$-axis. We have only the radial displacement $u_{r}$ which is independent of $\theta$ and $z$. In a generalized plane strain, we suppose that the planes perpendicular to the $z$-axis and $u_{r}$ is a function of the radial direction $r$ and the time $t$ only. The cylinder is placed in a constant primary magnetic field $H_{0}$. The medium is assumed to be non-ferromagnetic and ferroelectric. Neglecting the Thompson effect, the simplified Maxwell's equations of electro-dynamics for perfectly conducting elastic medium are:

$$
\nabla \times \vec{h}=\vec{j}, \quad \nabla \times \vec{E}=-\eta \frac{\partial \vec{h}}{\partial t}, \quad \nabla \cdot \vec{h}=0, \nabla \cdot \vec{E}=0,
$$

where

$$
\vec{E}=-\eta\left(\frac{\partial \vec{u}}{\partial t} \times \vec{H}\right), \quad \vec{h}=\nabla \times(\vec{u} \times \vec{H}),
$$

in which $\vec{H}$ is the magnetic field, $\vec{E}$ the electric field, $\vec{j}$ the current density, $\vec{u}$ the mechanical displacement, and $\vec{h}$ is the perturbed magnetic.

The material properties of the FGM cylinder are assumed to be function of the volume fraction of the constituent materials. The functionally graded between the physical properties and the radial direction $r$ for ceramic and metal FG cylinder is given by

$$
P(r)=P_{m} \mathrm{e}^{N_{P}\left(\frac{r-a}{b}\right)}, N_{P}=\frac{b}{b-a} \ln \left(\frac{P_{c}}{P_{m}}\right),
$$

where $P_{c}$ and $P_{m}$ are the corresponding properties of ceramic (outer surface) and metal (inner surface), respectively.

The equations of motion in the absence of the body force are

$$
\sigma_{i j, j}+\bar{\sigma}_{i j, j}=\rho(r) \ddot{u}_{i},
$$

where $\rho$ is the material density of the cylinder and it is also considered to be a function of $r$. The symbol ()$_{, j}$ means differentiation with respect to $x_{j}$. The mechanical stress tensor $\sigma_{i j}$ and Maxwell's electromagnetic stress tensor $\bar{\sigma}_{i j}$ are given, respectively, by

$$
\begin{aligned}
& \sigma_{i j}=\left(\lambda e_{i i}-\gamma T_{1}\right) \delta_{i j}+2 \mu e_{i j}, \\
& \bar{\sigma}_{i j}=\eta\left(h_{i} H_{j}+h_{j} H_{i}-h_{k} H_{k} \delta_{i j}\right),
\end{aligned}
$$

where $\delta_{i j}$ is Kronecker's delta, $T_{1}$ the absolute temperature, $\lambda$ and $\mu$ Lamé's coefficients, $\eta$ the magnetic permeability, $\gamma=(3 \lambda+2 \mu) \alpha$ the stress temperature modulus, in which $\alpha$ is the linear thermal expansion, and $e_{i j}$ is the stain tensor,

$$
e_{i j}=\frac{1}{2}\left(u_{i, j}+u_{j, i}\right) \text {. }
$$

For the present problem, considering the radial vibration of the medium, the only non-zero displacement is $u_{r}(r, t)$, so that

$$
e_{r r}=\frac{\partial u_{r}}{\partial r}, e_{\theta \theta}=\frac{u_{r}}{r}, e_{z z}=0 .
$$

Applying an initial magnetic field vector $\vec{H} \equiv\left[0,0, H_{0}\right]$ in cylindrical polar coordinate $(r, \theta, z)$ to Eqs.1 and 2, the field components in the medium are then obtained as

$$
\begin{aligned}
\vec{E} & \equiv-\eta\left[0,-H_{0} \frac{\partial u_{r}}{\partial t}, 0\right], \vec{h} \equiv\left[0,0,-H_{0}\left(\frac{\partial u_{r}}{\partial t}+\frac{u_{r}}{r}\right)\right], \\
\vec{j} & \equiv\left[0,-\frac{\partial h_{z}}{\partial r}, 0\right] .
\end{aligned}
$$

The magneto-elasto-dynamic equation, Eq.4, in the radial direction of the FG hollow cylinder is given by

$$
\frac{\partial \sigma_{r r}}{\partial r}+\frac{1}{r}\left(\sigma_{r r}-\sigma_{\theta \theta}\right)+f_{r}=\rho(r) \frac{\partial^{2} u_{r}}{\partial t^{2}},
$$

where

$$
f_{r}=\frac{\partial \bar{\sigma}_{r r}}{\partial r},
$$

is defined as Lorentz's force, and

$$
\begin{gathered}
\sigma_{r r}=\lambda(r)\left(\frac{\partial u_{r}}{\partial r}+\frac{u_{r}}{r}\right)+2 \mu(r) \frac{\partial u_{r}}{\partial r}-\gamma(r) T_{1}, \\
\sigma_{\theta \theta}=\lambda(r)\left(\frac{\partial u_{r}}{\partial r}+\frac{u_{r}}{r}\right)+2 \mu(r) \frac{u_{r}}{r}-\gamma(r) T_{1}, \\
\bar{\sigma}_{r r}=\eta(r) H_{0}^{2}\left(\frac{\partial u_{r}}{\partial r}+\frac{u_{r}}{r}\right) .
\end{gathered}
$$

The heat conduction equation in the presence of heat sources can be written in the form

$$
\left[\frac{\partial^{2}}{\partial r^{2}}+\left(\frac{1}{\kappa_{1}(r)} \frac{\mathrm{d} \kappa_{1}}{\mathrm{~d} r}+\frac{1}{r}\right) \frac{\partial}{\partial r}-\frac{1}{\kappa_{1}(r)} \frac{\partial}{\partial t}\right] T_{1}=-\frac{Q(r)}{\kappa_{2}(r)},
$$

where $\kappa_{1}$ is the thermal diffusivity, $\kappa_{2}$ the thermal conductivity, and $Q$ is the intensity of the applied heat source.

Generally, this study assumes that $\lambda, \mu, \gamma, \alpha$, $\kappa_{1}, \kappa_{2}, \eta$ and $\rho$ of the FG cylinder change continuously through the radial direction of the cylinder and obey the gradation relation given in Eq.3. 


\section{SOLUTION OF THE PROBLEM}

Introducing the following dimensionless variables may be simplifying the solving process:

$$
\begin{aligned}
& R=\frac{r}{b}, A=\frac{a}{b}, \tau=\frac{t}{b} \sqrt{\frac{\lambda_{m}}{\rho_{m}}}, \bar{T}=\frac{T_{1}(r, t)}{\rho_{m} T_{0}}, U=\frac{u_{r}(r, t)}{b \rho_{m} T_{0}}, \\
& \left(\sigma_{11}, \sigma_{22}\right)=\frac{\mathrm{e}^{N_{\lambda} A}}{\lambda_{m} \rho_{m} T_{0}}\left(\sigma_{r r}, \sigma_{\theta \theta}\right), \quad \Sigma_{11}=\frac{\mathrm{e}^{N_{\eta} A} \bar{\sigma}_{r r}}{H_{0}^{2} \eta_{m} \rho_{m} T_{0}}
\end{aligned}
$$

where $T_{0}$ is the reference temperature. In what follows we assume that the intensity of the applied heat source is given by the following form

$$
Q=\frac{\varepsilon \tau}{R} \mathrm{e}^{-\beta R},
$$

where $\beta$ being a non-negative constant, $\tau$ is dimensionless time and $\varepsilon$ is a constant. The effect of material properties variation of the FG cylinder can be taken into account in Eqs.9 and 14. The substitution of Eqs.10-12 into Eqs.9 and 14 with the aid of the dimensionless variables given in Eq.15 produces the governing equations for the FG cylinder as follows:

$$
\begin{aligned}
& \left(1+\bar{\eta}_{m} H_{0}^{2} \mathrm{e}^{N_{\eta \lambda} R}+2 \bar{\mu}_{m} \mathrm{e}^{N_{\mu \lambda} R}\right)\left[R^{2} \frac{\partial^{2} U}{\partial R^{2}}+R \frac{\partial U}{\partial R}-U\right] \\
& +R^{2}\left(N_{\lambda}+\bar{\eta}_{m} N_{\eta} H_{0}^{2} \mathrm{e}^{N_{\eta \lambda} R}+2 \bar{\mu}_{m} N_{\mu} \mathrm{e}^{N_{\mu \lambda} R}\right) \frac{\partial U}{\partial R} \\
& +R\left(N_{\lambda}+\bar{\eta}_{m} N_{\eta} H_{0}^{2} \mathrm{e}^{N_{\eta \lambda} R}\right) U-\bar{\gamma}_{m} R^{2} \mathrm{e}^{N_{\gamma \lambda} R}\left[\frac{\partial \bar{T}}{\partial R}+N_{\gamma} \bar{T}\right] \\
& =R^{2} \mathrm{e}^{N_{\rho \lambda}(R-A)} \frac{\partial^{2} U}{\partial \tau^{2}}, \\
& {\left[\frac{\partial^{2}}{\partial R^{2}}+\left(N_{\kappa_{1}}+\frac{1}{R}\right) \frac{\partial}{\partial R}-\alpha_{1} \mathrm{e}^{-N_{\kappa_{1}} R} \frac{\partial}{\partial \tau}\right] \bar{T}=-\alpha_{2} \frac{\tau}{R} \mathrm{e}^{-\left(\beta+N_{\kappa_{2}}\right) R},}
\end{aligned}
$$

where

$$
\begin{gathered}
\bar{\xi}_{m}=\frac{\xi_{m}}{\lambda_{m} \mathrm{e}^{N_{\xi \lambda} A}}, \quad N_{\xi \zeta}=N_{\xi}-N_{\zeta}, \\
\alpha_{1}=\frac{b \mathrm{e}^{N_{K_{1}} A}}{\kappa_{1 m}} \sqrt{\frac{\lambda_{m}}{\rho_{m}}}, \quad \alpha_{2}=\frac{b^{2} \varepsilon \mathrm{e}^{N_{\kappa_{2}} A}}{T_{0} \rho_{m} \kappa_{2 m}} .
\end{gathered}
$$

Note that, $\lambda_{m}, \mu_{m}, \gamma_{m}, \kappa_{1 m}, \kappa_{2 m}, \eta_{m}$ and $\rho_{m}$ are Lamé's constants, thermal modulus, thermal diffusivity, thermal conductivity, magnetic permeability, and density of the homogeneous metal material, respectively.

The dimensionless stresses induced by the temperature $\bar{T}$ and the electromagnetic stress are related to the dimensionless radial displacement $U$ by

$$
\begin{gathered}
\sigma_{11}=\mathrm{e}^{N_{\lambda} R}\left(\frac{\partial U}{\partial R}+\frac{U}{R}\right)+2 \bar{\mu}_{m} \mathrm{e}^{N_{\mu} R} \frac{\partial U}{\partial R}-\bar{\gamma}_{m} \mathrm{e}^{N_{\gamma} R} \bar{T}, \\
\sigma_{22}=\mathrm{e}^{N_{\lambda} R}\left(\frac{\partial U}{\partial R}+\frac{U}{R}\right)+2 \bar{\mu}_{m} \mathrm{e}^{N_{\mu} R} \frac{U}{R}-\bar{\gamma}_{m} \mathrm{e}^{N_{\gamma} R} \bar{T}, \\
\Sigma_{11}=\mathrm{e}^{N_{\eta} R}\left(\frac{\partial U}{\partial R}+\frac{U}{R}\right) .
\end{gathered}
$$

The elastic solution for the FG hollow cylinder is completed by the application of the initial and boundary conditions. The initial conditions can be expressed as

$$
\bar{T}=0, \quad U=\frac{\partial U}{\partial \tau}=0 \quad \text { at } \quad \tau=0 .
$$

The boundary conditions at the inner and outer radii of the FG cylinder may be expressed as

$$
\begin{array}{llll}
\bar{T}=0, & U=0 \quad \text { at } & R=A, \\
\frac{\partial \bar{T}}{\partial R}=0, & U=0 \quad \text { at } & R=1 .
\end{array}
$$

\section{NUMERICAL SCHEME}

A finite element scheme is used here to get the temperature and radial displacement. The finite difference grids with spatial intervals $h$ in the radial direction and $k$ as the time step, and use the subscripts $i$ and $n$ to denote the $i$ th discrete point in the $R$ direction and the $n$th discrete time. A mesh is defined by

$$
\begin{array}{ll}
R_{i}=A+i h, & i=0,1,2, \ldots, J, \\
\tau^{n}=k n, & n=0,1,2,3, \ldots .
\end{array}
$$

The displacement and temperature may be given at any nodal location by

$$
U\left(R_{i}, \tau^{n}\right)=U_{i}^{n}, \quad \bar{T}\left(R_{i}, \tau^{n}\right)=\bar{T}_{i}^{n} .
$$

The equation of motion and the heat conduction equation, given in Eqs.17 and 18, may be expressed in the finite difference as

$$
\begin{aligned}
& U_{i}^{n+1}=f_{1} U_{i+1}^{n}+2\left(1-f_{2}\right) U_{i}^{n}+f_{3} U_{i-1}^{n}- \\
& U_{i}^{n-1}+f_{4}\left(\bar{T}_{i+1}^{n}+2 h N_{\gamma} \bar{T}_{i}^{n}-\bar{T}_{i-1}^{n}\right),
\end{aligned}
$$

$$
\bar{T}_{i}^{n+1}=g_{1} \bar{T}_{i+1}^{n}+\left(1-4 g_{2}\right) \bar{T}_{i}^{n}+g_{3} \bar{T}_{i-1}^{n}+g_{4},
$$

where

$$
\begin{aligned}
& {\left[f_{1}, f_{2}, f_{3}\right]=\frac{k^{2}}{2 V^{2} h^{2}(A+i h)} \mathrm{e}^{-N_{\rho \lambda} i h}\left[\overline{f_{1}}, \frac{f_{2}}{A+i h}, \overline{f_{3}}\right]} \\
& f_{4}=-\frac{k^{2} \bar{\gamma}_{m}}{2 V^{2} h} \mathrm{e}^{N_{\gamma \lambda} A} \mathrm{e}^{N_{\gamma \rho} i h}, \\
& g_{s}=\frac{k}{2 \alpha_{1} h^{2}} \mathrm{e}^{N_{\kappa_{1}}(A+i h)} \bar{g}_{s}, \\
& g_{4}=\frac{k^{2} \alpha_{2} h}{\alpha_{1}(A+i h)} \mathrm{e}^{\left(N_{\kappa_{1}}-N_{\kappa_{2}}-\beta\right)(A+i h)}, \quad s=1,2,3
\end{aligned}
$$


in which

$$
\begin{gathered}
\bar{f}_{1}=h+(A+i h)\left(2+h N_{\lambda}\right)+ \\
2 \bar{\mu}_{m} \mathrm{e}^{N_{\mu \lambda}(A+i h)}\left[h+(A+i h)\left(2+h N_{\mu}\right)\right] \\
+\bar{\eta}_{m} H_{0}^{2} \mathrm{e}^{N_{\eta \lambda}(A+i h)}\left[h+(A+i h)\left(2+h N_{\eta}\right)\right], \\
\bar{f}_{2}=2(A+i h)^{2}+h^{2}\left[1-N_{\lambda}(A+i h)\right]+ \\
2 \bar{\mu}_{m} \mathrm{e}^{N_{\mu \lambda}(A+i h)}\left[2(A+i h)^{2}+h^{2}\right] \\
+\bar{\eta}_{m} H_{0}^{2} \mathrm{e}^{N_{\eta \lambda}(A+i h)}\left\{2(A+i h)^{2}+h^{2}\left[1-N_{\eta}(A+i h)\right]\right\}, \\
\bar{f}_{3}=h+(A+i h)\left(2-h N_{\lambda}\right)+ \\
2 \bar{\mu}_{m} \mathrm{e}^{N_{\mu \lambda}(A+i h)}\left[h+(A+i h)\left(2-h N_{\mu}\right)\right] \\
+\bar{\eta}_{m} H_{0}^{2} \mathrm{e}^{N_{\eta \lambda}(A+i h)}\left[h+(A+i h)\left(2-h N_{\eta}\right)\right], \\
\bar{g}_{1}=2+h\left(N_{\kappa_{1}}+\frac{1}{A+i h}\right), \bar{g}_{2}=1, \\
\bar{g}_{3}=2-h\left(N_{\kappa_{1}}+\frac{1}{A+i h}\right) .
\end{gathered}
$$

\section{NUMERICAL RESULTS}

The above finite element scheme is used here to get the temperature and radial displacement through the radial direction of the FGM hollow cylinder. The least square method is used also to get the appropriate stresses in the FGM hollow cylinder. The results are presented in the non-dimensional form:

$$
\tau^{*}=\tau \times 10^{4}, T=\bar{T} \times 10^{17}, u=U \times 10^{19}, \sigma=\sigma_{11} \times 10^{11} .
$$

All results of this article are for aluminum as inner metal surface and alumina as outer ceramic surface. Generally, the magnetic permeability $\eta_{p}=\eta_{0} K_{p} \quad(p=$ $m$ or $c$ ) is given in terms of the permeability of space $\eta_{0}=4 \pi \times 10^{-7} \mathrm{~N} / \mathrm{A}^{2}$ and the relative permeability for both aluminum $K_{m}$ and alumina $K_{c}$. The material properties are assumed to be as:

\section{metal (aluminum):}

$$
\begin{aligned}
& E_{m}=70 \mathrm{GPa}, v_{m}=0.35, \rho_{m}=2700 \mathrm{Kg} / \mathrm{m}^{3}, K_{m}=2.3, \\
& \alpha_{m}=23.1 \times 10^{-6} / \mathrm{K}, \kappa_{1 m}=84.18 \times 10^{-6} \mathrm{~m}^{2} / \mathrm{s}, \\
& \kappa_{2 m}=237 \mathrm{~W} /(\mathrm{m} \mathrm{K}) .
\end{aligned}
$$

\section{ceramic (alumina):}

$$
\begin{gathered}
E_{c}=116 \mathrm{GPa}, v_{c}=0.33, \rho_{c}=3000 \mathrm{Kg} / \mathrm{m}^{3}, K_{c}=1.0, \\
\alpha_{c}=8.7 \times 10^{-6} / \mathrm{K}, \kappa_{1 m}=1.06 \times 10^{-6} \mathrm{~m}^{2} / \mathrm{s}, \\
\kappa_{2 m}=1.78 \mathrm{~W} /(\mathrm{m} \mathrm{K}) .
\end{gathered}
$$

Note that the properties of $\lambda, \mu$, and $\gamma$ for metal or ceramic are graded through the radial direction according to the following relations:

$$
\begin{aligned}
& \lambda_{p}=\frac{E_{p} v_{p}}{\left(1+v_{p}\right)\left(1-2 v_{p}\right)}, \quad \mu_{p}=\frac{E_{p}}{2\left(1+v_{p}\right)}, \\
& \gamma_{p}=\left(3 \lambda_{p}+2 \mu_{p}\right) \alpha_{p}, \quad(p=m, c) .
\end{aligned}
$$

Results are presented in Figures 1-6 for temperature, radial displacement and redial stress according to the fixed constants

$$
\varepsilon=0.25, A=0.2, T_{0}=20 \mathrm{~K}, H_{0}=10^{6} \text { Oersted. }
$$

The sensitivity of the time parameter $\tau *$ and the

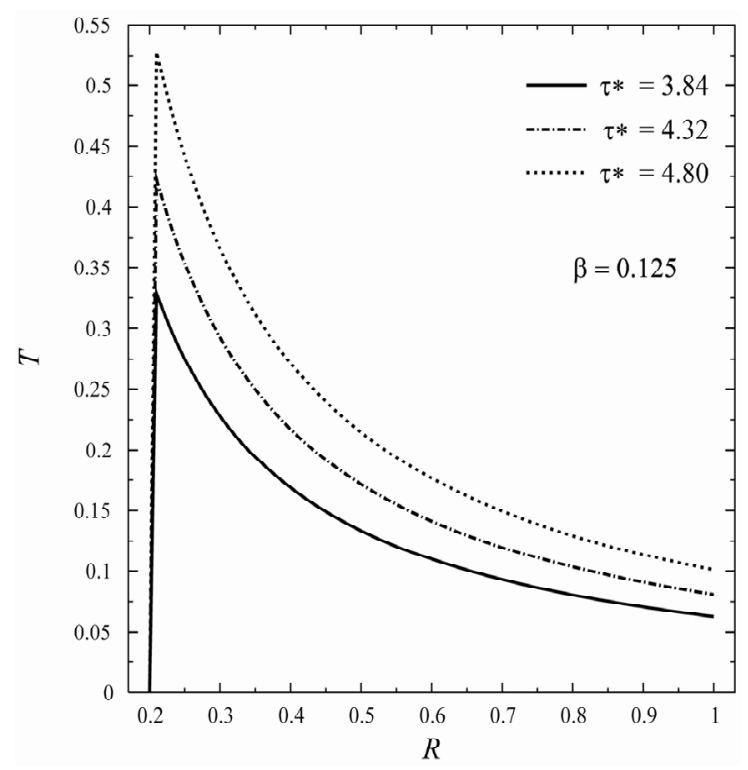

Figure 1. Variation of temperature $T$ through the radial direction of the FGM hollow cylinder at different times for $\beta=0.125$.

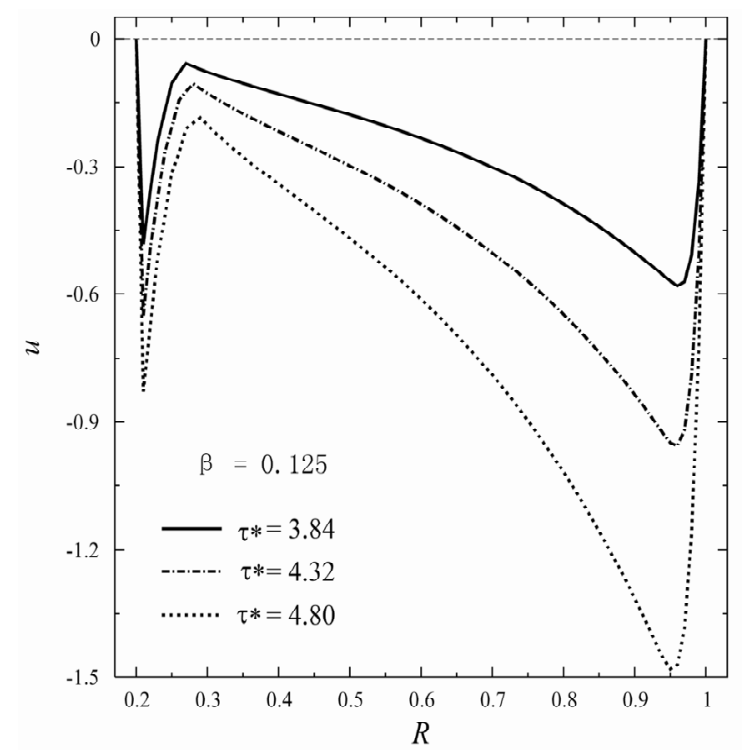

Figure 2. Variation of radial displacement $U$ through the radial direction of the FGM hollow cylinder at different times for $\beta=0.125$. 


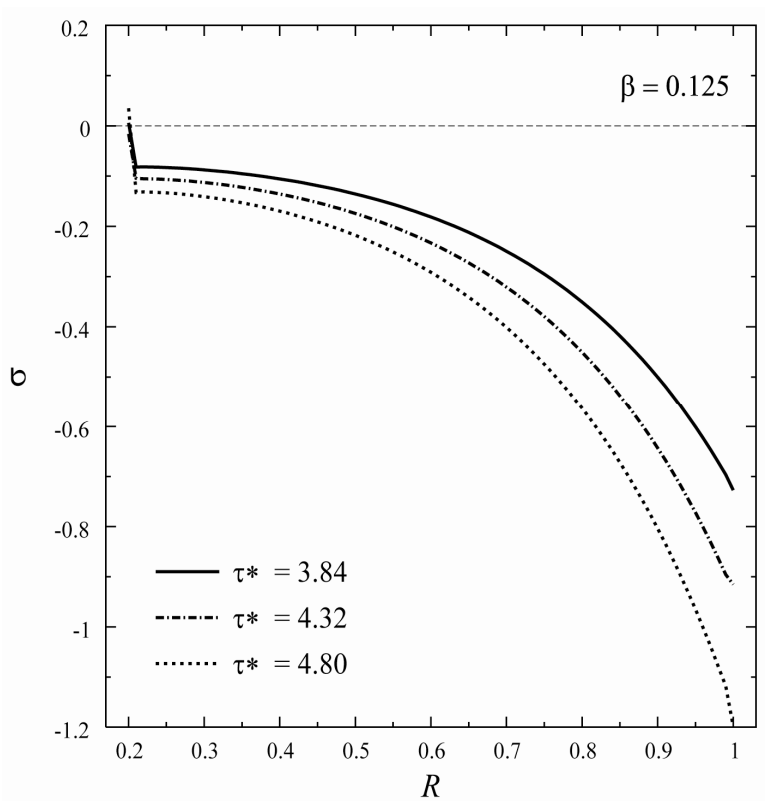

Figure 3. Variation of radial stress $\sigma$ through the radial direction of the FGM hollow cylinder at different times for $\beta$ $\sigma=0: 125$.

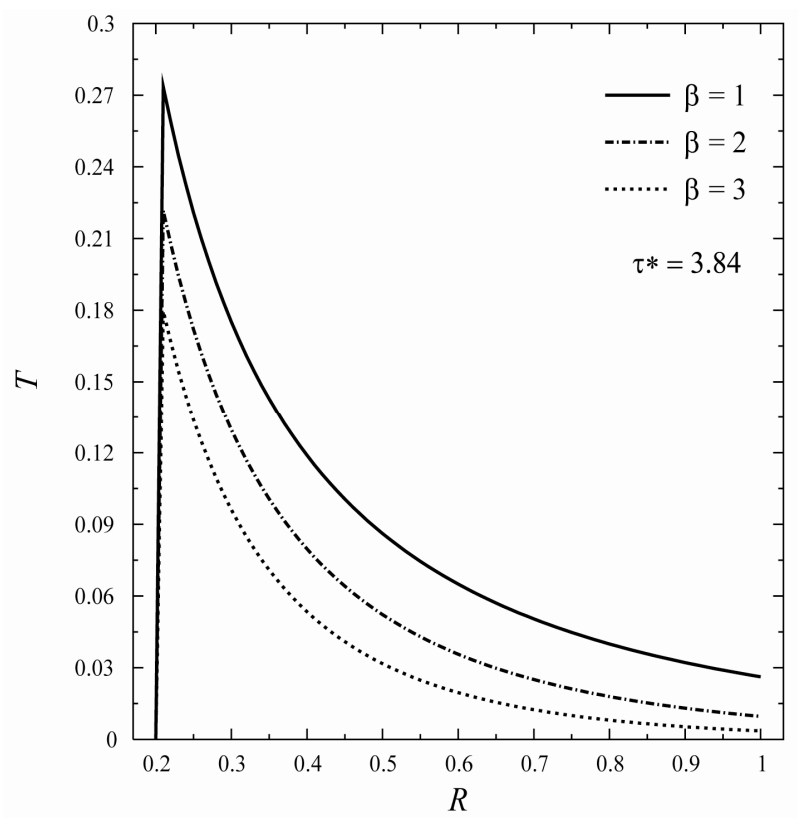

Figure 4. Variation of temperature $T$ through the radial direction of the FGM hollow cylinder at $\tau *=3.84$ for different values of $\beta$.

exponential factor $\beta$ given in c (16) for the heat source, are discussed here. The values of $\tau *$ and $\beta$ will be chosen through the illustration figures.

Figures 1-3 represent the variations of the dimensionless temperature $T$, radial displacement $u$, and radial stress $\sigma$ through the radial direction of the FGM hollow cylinder. Three values of the time parameter $\tau *$ are

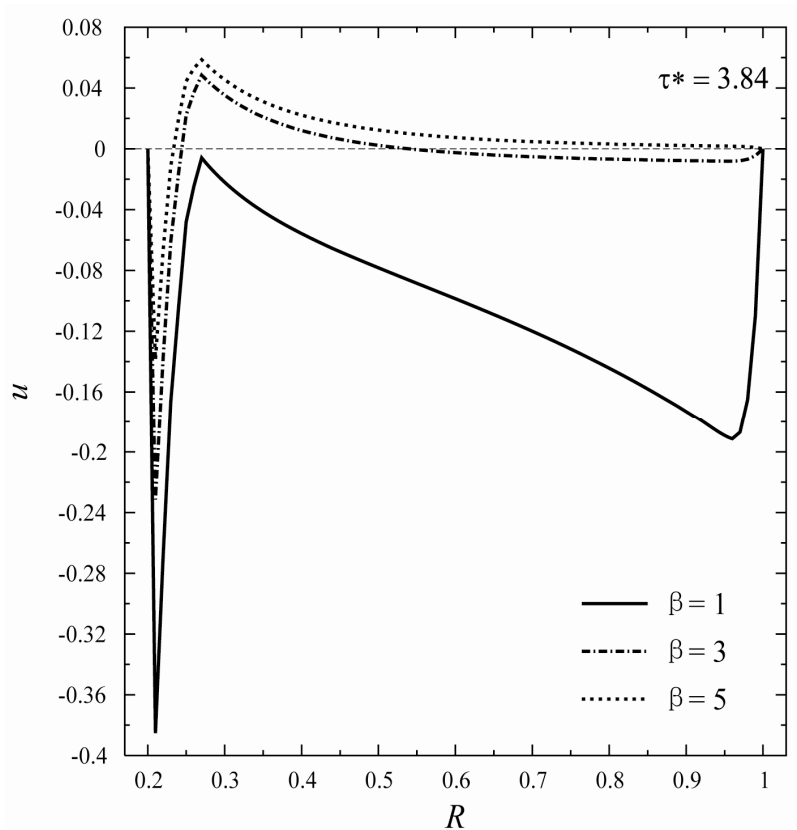

Figure 5. Variation of radial displacement $U$ through the radial direction of the FGM hollow cylinder at $\tau *=3.84$ for different values of $\beta$.

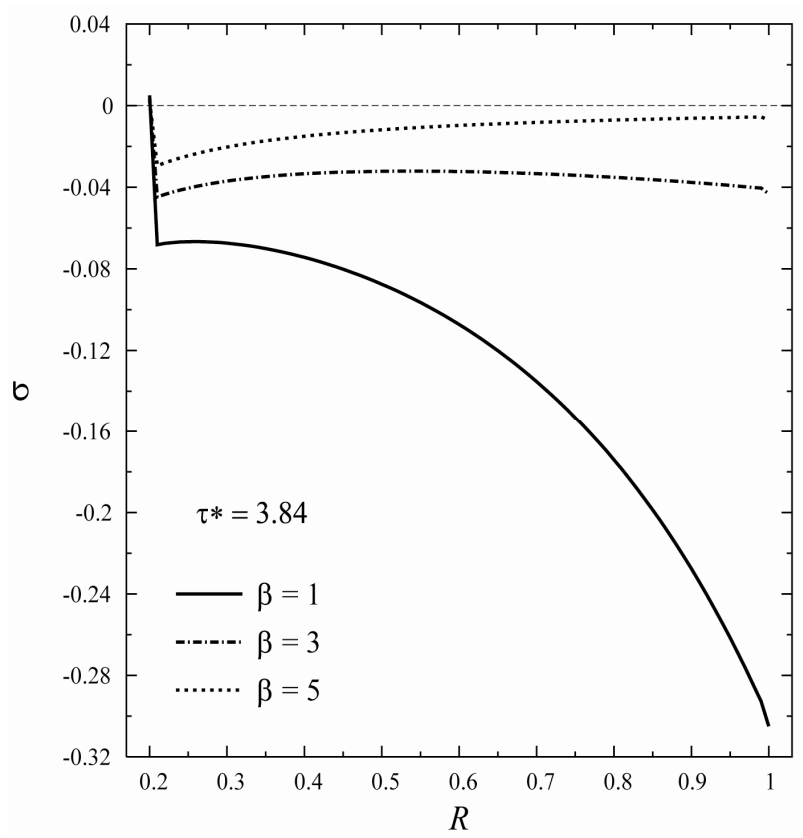

Figure 6. Variation of radial stress $\sigma$ through the radial direction of the FGM hollow cylinder at $\tau *=3: 84$ for different values of $\beta$.

used. Figure 1 shows that the temperature is sharply increasing to get its maximum at a neighborhood point of the inner metal surface. After that, it slightly decreases to reach a fixed value at the outer ceramic surface. The temperature decreases as $\tau *$ decreases. Fig- 
ure 2 shows the displacement distribution through the radial direction of the FGM cylinder. It is seen that the displacement decreases dramatically within a very small range of radial direction at first, and then it increases gradually to a local higher value. Once again, it decreases gradually to a minimum value near the outer surface. The displacement $u$ decreases as $\tau *$ increases. The distribution of radial stress $\sigma$ through the radial direction of the FGM cylinder is plotted in Figure 3 for different values of $\tau *$. It is seen that $\sigma$ decreases dramatically within a very small range of radial direction at first, and then it decreases gradually to its minimum value at the ceramic outer surface of the cylinder. Also, $\sigma$ decreases as $\tau *$ increases.

The effects of the heat source intensity coefficient $\beta$ on the temperature, displacement and stress at fixed time parameter $\tau^{*}=3.84$ are plotted in Figures 4-6. Figure 4 shows that for different $\beta$, the change tendencies of temperature appear in same obviously. The temperature increases as $\beta$ decreases. Figures 5 and 6 show that the higher values of heat source intensity coefficient $\beta$ have only a little effect on $u$ and $\sigma$. It is seen that the radial displacement and radial stress under larger $\beta$ are evidently different from that under small $\beta$.

\section{CONCLUSIONS}

The main contribution in this paper is to describe the effects of time parameter and heat source intensity of exponentially graded material cylinder on temperature, displacement and stresses. The results are very sensitive to the change of time and heat source through the radial direction of the cylinder. The solution method in this article may be used as a useful reference to investigate the temperature, radial displacement, radial and circumferential stresses, and electromagnetic stress in the cylinder. The results carried out can be used to predict the electro-magneto-thermoelastic response at different times and for different heat source intensities according to the engineering requirements.

\section{REFERENCES}

[1] Wang, X., Lu, G. and Guillow, S.R. (2002) Magneto- thermodynamic stress and perturbation of magnetic field vector in a solid cylinder. Journal of Thermal Stresses, 25, 909-926.

[2] X. Wang and H.L. Dai: Magnetothermodynamic stress and perturbation of magnetic field vector in an orthotropic thermoelastic cylinder. The International Journal of Engineering Science, 42, 539-556.

[3] Wang, X. and Dai, H.L. (2004) Magneto-thermo-dynamic stress and perturbation of magnetic field vector in a hollow cylinder. Journal of Thermal Stresses, 3, 269288.

[4] Dai, H.L. and Wang, X. (2006) Magneto-thermo-electro-elastic transient response in a piezoelectric hollow cylinder subjected to complex loadings. The International Journal of Solids and Structures, 43, 5628-5646.

[5] H.L. Dai and Fu, Y.M. (2007) Magnetothermoelastic interactions in hollow structures of functionally graded material subjected to mechanical loads. The International Journal of Pressure Vessels and Piping, 84, 132-138.

[6] Chen, C.K. and Yang, Y.C. (1986) Thermoelastic transient response of an infinitely long annular cylinder composed of two different materials. The International Journal of Engineering Science, 24, 569-581.

[7] Jane, K.C. and Lee, Z.Y. (1999) Thermoelastic transient response of an infinitely long multilayered cylinder. $\mathrm{Me}$ chanics Research Communications, 26, 709-718.

[8] Lee, Z.-Y. (2004) Hybrid numerical method applied to 3-D multilayers hollow cylinder with time-dependent boundary conditions. Applied Mathematics and Computation, 150, 25-43.

[9] Awaji, H. and Sivakuman, R. (2001) Temperature and stress distributions in a hollow cylinder of functionally graded material: the case of temperature-dependent material properties. Journal of the American Ceramic Society, 84, 1059-1065.

[10] Yang, Y.-C., Chu, S.-S., Lee, H.-L. and Lin, S.-L. (2006) Hybrid numerical method applied to transient hygrothermal analysis in an annular cylinder. International Communications in Heat and Mass Transfer, 33, 102-111.

[11] Chen, Y.Z. (2000) Stress intensity factors in a finite length cylinder with a circumferential crack. International Journal of Pressure Vessels and Piping, 77, 439444.

[12] Jane, K.C. and Lee, Z.Y. (1999) Thermoelastic transient response of an infinitely long annular multilayered cylinder. Mechanics Research Communications, 26, 709718. 